\title{
Demo: 3-D Wireless Charging for Indoor Electronics Using Multimode Quasistatic Cavity Resonators
}

Takuya Sasatani

The University of Tokyo

JSPS Research Fellow

sasatani@akg.t.u-tokyo.ac.jp

Alanson P. Sample

University of Michigan

apsample@umich.edu

Yoshihiro Kawahara

The University of Tokyo

kawahara@akg.t.u-tokyo.ac.jp
Permission to make digital or hard copies of part or all of this work for personal or classroom use is granted without fee provided that copies are not made or distributed for profit or commercial advantage and that copies bear this notice and the full citation on the first page. Copyrights for third-party components of this work must be honored. For all other uses, contact the owner/author(s).

Copyright held by the owner/author(s).

UbiComp/ISWC'18 Adjunct, October 8-12, 2018, Singapore, Singapore ACM 978-1-4503-5966-5/18/10.

https://doi.org/10.1145/3267305.326757

\begin{abstract}
Most existing wireless power transfer (WPT) solutions are limited to 2-D configurations, which limits mobility when charging electronics. What is needed are 3-D WPT, which can deliver power anywhere in large volumes (e.g., factories, rooms, toolbox, etc). WPT using quasistatic cavity resonators (QSCR) proposed a route towards truly ubiquitous WPT, which safely charges devices as they enter a WPT enabled space. However, several drawbacks exist to this approach such as the need for a central pole and the spatially non-uniform power availability. To address these issues, we demonstrate a WPT system based on "multimode" QSCR [4]; this structure possess two resonant modes: pole dependent $(\mathrm{PD})$ mode, which resembles the previous QSCR work and pole independent $(\mathrm{PI})$ mode, which works whether or not the pole exist. This structure enables two operations: (i) pole-less operation, which works to the same degree as the previous QSCR without the central pole and (ii) dual-mode operation, which although requires the central pole, enables high-efficiency WPT all over the volume.
\end{abstract}

\section{Author Keywords}

Wireless Power Transfer; Ubiquitous Computing

\section{ACM Classification Keywords}

H.5.m [Information interfaces and presentation (e.g., $\mathrm{HCl})]$ : Miscellaneous 


\section{Related Works}

The Qi standard brought inductive WPT into commerce, although this technology only enables power delivery to a single specific point (i.e. on a cradle) [2]. WPT via magnetic resonant coupling extended the transfer distance and improved misalignment tolerance of inductive WPT, although the power delivery range is still restricted to 2-D surfaces [3]. Recently, a new route towards ubiquitous WPT was proposed using quasistatic cavity resonators (QSCR), which is a metallic cavity containing a central pole with capacitors inserted in a gap in the pole; this generates a 3-D magnetic field distribution within the cavity and enables 3-D WPT [1]. Although QSCR showed promising features for 3-D WPT, it is known that this method withholds two critical drawbacks; the need for a conductive pole in the middle of the cavity and the spatially non-uniform power availability. "Multimode" QSCR, which we demonstrate here is an new class of QSCR, which addresses the aforementioned challenges by utilizing the multiple resonant modes [4].

\section{The Structure of Multimode QSCR}

The structure of the demonstrated multimode QSCR is shown in Figure 1(a,b). The simulated current and magnetic field of the two resonant modes are illustrated in figure 2; it can be seen that the magnetic field of these two modes cover the weak area of each other. The structure is implemented by bolting on lumped capacitors on sheet metal. The geometrical parameters referring to Figure 1 is $w=600, h=300, p=0.5, g=10, d_{p}=19$ (unit: $\mathrm{mm}$ ), whereas the values of the capacitors are $C_{1}=3.26 \mathrm{nF}$, $C_{2}=5.8 \mathrm{nF}$. The power is input to the multimode QSCR via the drive coil [See figure 3 (a)], which is inductively coupled to the resonant modes of the cavity. This stimulates the resonant modes of the multimode-QSCR and generates a magnetic field that permeates the cavity volume; power can be supplied to receivers via this magnetic field.
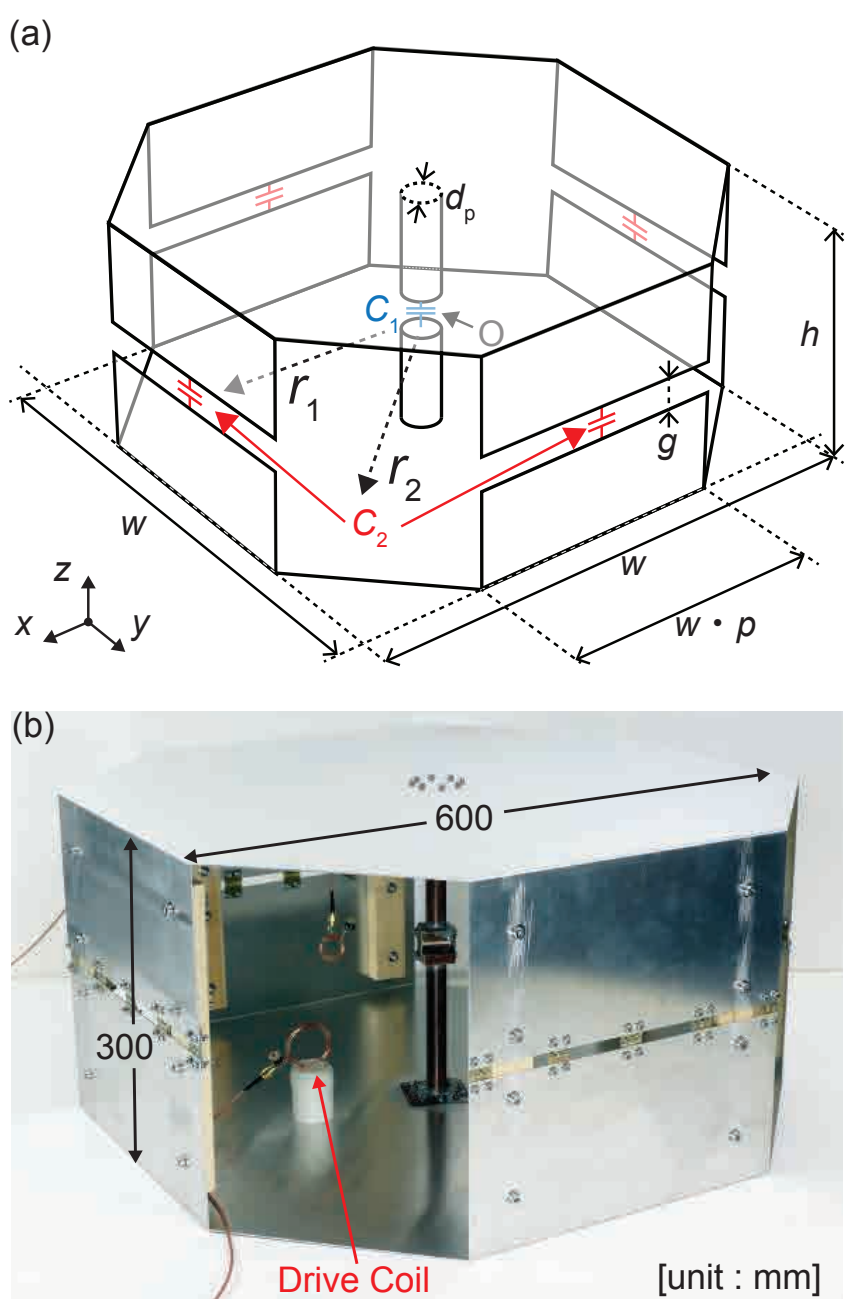

Figure 1: (a) Geometry of the demonstrated multimode QSCR. $r_{1}$ and $r_{2}$ show the series of positions where the RX was placed on for evaluation of efficiency [See. figure 2(c,d).] (b) The implemented multimode QSCR. 


\section{Evaluation of System Performance}

The power transfer efficiency was evaluated through FEM simulations and measurements. A 10 turn, $10 \mathrm{~mm}$ radius, circular receiver coil with a Q-factor of 100 was used as the target of power delivery. Note that the area of this receiver is smaller than $10^{-3}$ of the cross-sectional area of the WPT enabled volume. The simulated efficiency was calculated using coupling mode theory (CMT) [1]. As for measured efficiency, the maximum achievable efficiency, calculated from the measured S-parameters was used [5]. The measured and simulated WPT efficiency, evaluated on line $r_{1}$ and $r_{2}$ in figure 1 (a) are plotted in figure 2 (c,d). Moreover, by performing simulation-based evaluation in the full cavity volume, an average WPT efficiency of $32.8 \%$ (pole-less operation) and $40.5 \%$ (dual-mode operation) was obtained.

\section{Applications and Demonstrations}

The potential applications of our multimode QSCR-based 3D WPT system ranges from toolboxes that reanimate electric tools with dead batteries, to factories with hundreds of wireless charged, maintenance-free Internet of Things (IoT) nodes. To show one use-case, a prototype of a miniaturized wireless powered room is shown in figure $3(\mathrm{~b})$. This demonstration was conducted with the central pole taken off and all the LEDs are powered wirelessly; they represent the devices to be charged.

\section{Conclusion}

In this study, we demonstrated a multimode QSCR-based 3-D WPT system through a miniaturized WPT enabled room scenario; this is a new approach towards ubiquitous WPT. In addition, our simulations and measurements showed that an average efficiency over $40 \%$ can be obtained throughout the full cavity volume; the notable point here that the receiver we used is smaller than $10^{-3}$ of the cross-section of the WPT enabled volume. (a) Pole Dependent (PD) Mode

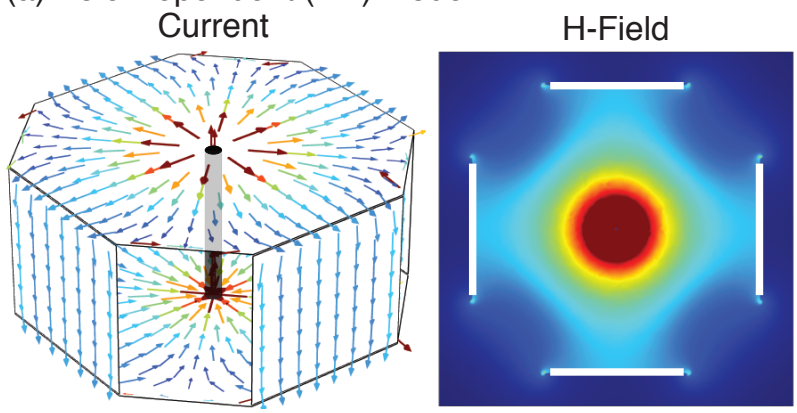

(b) Pole Independent (PI) Mode
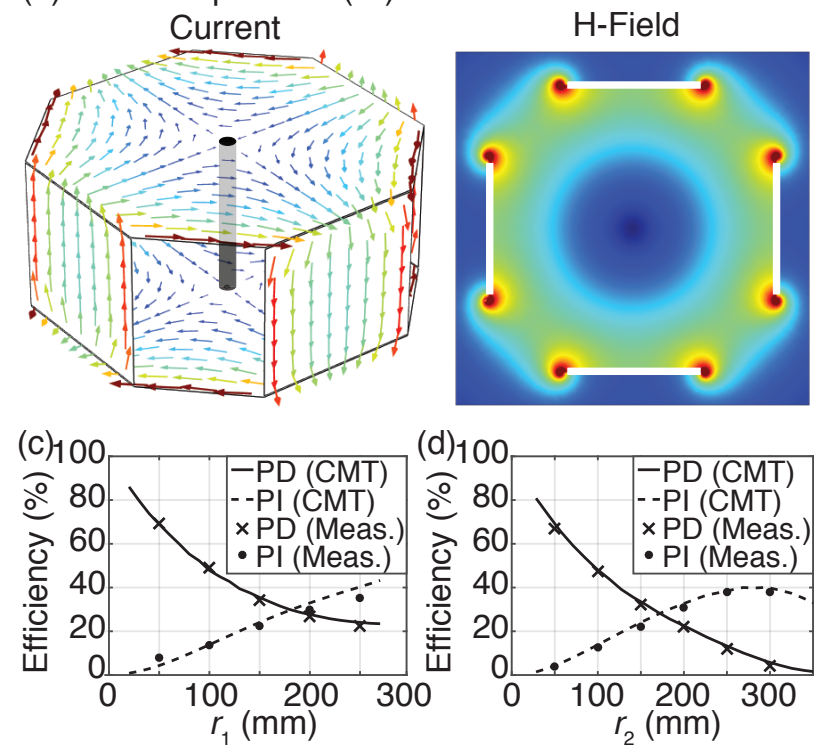

Figure 2: $(a, b)$ The simulated current and magnetic field distribution of (a) Pole dependent (PD) mode and (b) Pole independent (PI) mode. (c,d) The WPT efficiency when a $20 \mathrm{~mm}$ diameter $\mathrm{RX}$ is placed on lines $r_{1}$ and $r_{2}$ [See figure $1(\mathrm{a})$ ]. 
(a)

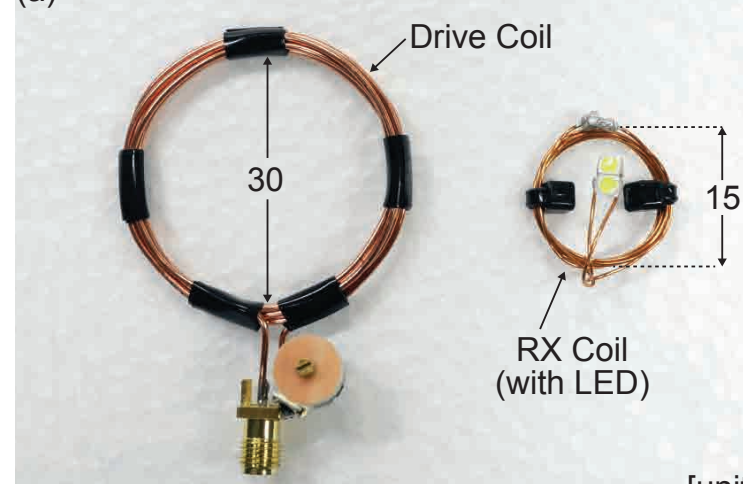

[unit : mm]

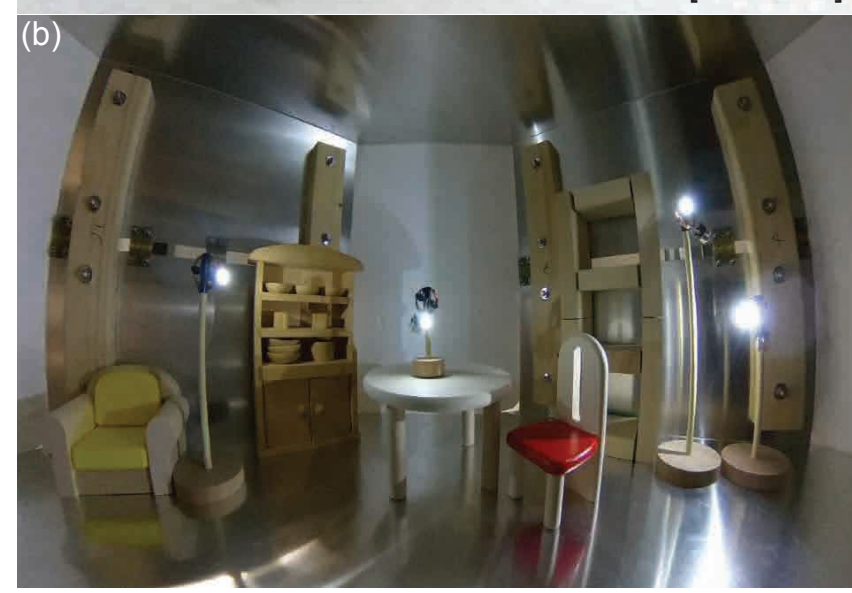

Figure 3: (a) The drive coil and the wireless powered LED.

(b) Demonstration of the miniaturized WPT system seen from inside the cavity. The wireless powered LEDs are the only light sources in this image.

\section{Acknowledgements}

This work was supported by a Grant-in-Aid for JSPS Fellows JP18J22537 and JST ERATO Grant Number JPMJER1501.

\section{REFERENCES}

1. Matthew J Chabalko, Mohsen Shahmohammadi, and Alanson P Sample. 2017. Quasistatic Cavity Resonance for Ubiquitous Wireless Power Transfer. PloS ONE 12, 2 (February 2017), e0169045. DOI : http://dx.doi.org/10.1371/journal.pone.0169045

2. Shu Yuen Ron Hui, Wenxing Zhong, and Chi Kwan Lee. 2014. A critical review of recent progress in mid-range wireless power transfer. IEEE Transactions on Power Electronics 29, 9 (September 2014), 4500-4511. DOI :

http://dx.doi.org/10.1109/TPEL. 2013.2249670

3. Andre Kurs, Aristeidis Karalis, Robert Moffatt, John D Joannopoulos, Peter Fisher, and Marin Soljačić. 2007. Wireless power transfer via strongly coupled magnetic resonances. science 317, 5834 (2007), 83-86. DOI : http://dx.doi.org/10.1126/science. 1143254

4. Takuya Sasatani, Matthew J Chabalko, Yoshihiro Kawahara, and Alanson P Sample. 2017. Multimode Quasistatic Cavity Resonators for Wireless Power Transfer. IEEE Antennas Wireless Propagation Letters 16 (August 2017), 2746-2749. DOI :

http://dx.doi.org/10.1109/LAWP.2017.2744658

5. Meysam Zargham and P Glenn Gulak. 2012. Maximum achievable efficiency in near-field coupled

power-transfer systems. IEEE Transactions on Biomedical Circuits and Systems 6, 3 (January 2012), 228-245. DOI :

http://dx.doi.org/10.1109/TBCAS.2011.2174794 